\title{
On the Ideological Management of Sailors
}

\author{
Chong Zhao*, Lihong Du, Zengji Wei
}

Xi 'an Institute of High Technology, Xi'an, China

${ }^{*}$ Corresponding author. Email: zc951118@163.com

\begin{abstract}
This paper elaborates the ideological issues confronted by the crew in our country under the circumstances of sailing missions. It also analyses the current situation they are faced with from three perspectives: laws and regulations, personnel structure, and ideology, and proposes some coping management tactics based on the analysis. These tactics can be applied to release crew's negative psychological emotion in time, and help them maintain a high morale all the time, so that the ideological management of the crew can be promoted in the sailing mission situations.
\end{abstract}

Keywords: Mariner, Sailing, Ideological management.

\section{远航船员思想管理探析}

\author{
赵独”, 杜荔红, 魏增基
}

西安高技术研究所讲师, 西安, 中国

*通讯作者. 邮箱: zc951118@163.com

\section{中文摘要}

本文阐述了我国远航船员在长期远洋航行环境下面临的思想问题，从法规制度，人员结构，意识形态等方面分 析我国船员所面临的现状，并在此基础上提出针对性的管理方法，及时疏解船员负面心理情绪，始终保持高扬 士气，以此提高我国船员长期海上环境下的人员思想管理状况。

关键词: 船员，远航，思想管理

\section{1. 当前我国远航船员思想情况综述}

随着我国对外贸易的逐渐增强, 远洋航运业承担 了越来越多的国际贸易任务, 而航行周期的增长, 如 何提升船员的思想管理水平显得越来越重要

\section{1. 船员负面思想主要有以下两种类型}

船员本身就是一个非常特殊的群体, 在长期出海 环境下更是极易发生负面思想问题，根据调查，大部 分船员在生理和心理上主要有以下标志性问题。

\subsection{1. 生理反应}

在调查中明显感觉到, 随着航行任务的顺利推进, 少数船员出现了逆反和烦躁情绪, 表现在对提出的各 项管理及纪律要求服从但不情愿。对上级管理、伙食 稍不满意就无限扩大发泄, 个别船员甚至出现言语肢 体冲突、人际关系紧张等问题。从船员日常就餐观察, 盘子里的菜越来越少了, 餐厨垃圾越来越多了, 分析 主要有两个方面原因: 一是航行所需的副食蔬菜大都 是起航前补给的。在航行任务一个多月后, 蔬菜基本 消耗殆尽。若靠泊海外只能在所靠泊国家采购补给, 但补给果蔬大都和国内同名而不同味，很多船员不习 惯口味。二是长期在外出海, 炊事人员也已经“黔驴 
技穷”, 船员对饭菜的味道从喜爱渐渐变为厌烦, 普 遍感觉到大锅饭“口味太淡, 没胃口”。食欲不振, 不 仅造成船员身体免疫力下降，而且容易引发更严重的 负面情绪。调查了解到, 在航行任务中后期, 船员睡 眠质量越来越差, 个别的甚至患上了严重的精神衰弱, 由于每个船员都有所属值班岗位, 昼夜颠倒成为常态, 商船内部照明通常都处于常亮状态, 船员接受不到自 然光线照射, 因此常常失眠到后半夜, 严重威胁到了 航行安全。

\subsection{2. 心理反应}

调查发现, 心理匮乏现象在大部分船员中普遍存 在。他们经常出现容易烦躁、易怒、情绪低迷等症状。 心理贵乏是指官兵在感觉、情感、信息等方面的匮乏 及其对心理状态造成的不良影响, 容易诱发过重心理 负担。调查中也发现, 各岗位船员所住舱室大都卫生 秩序不佳, 进一步发现他们大都是负责航行保障的人 员, 工作环境要么是高温度、高噪音、强震动的机舱, 要么是无聊的报房、罗经室。调查时, 他们表示, 长 期高噪音、固定音频环境使其对声音不敏感, 对外界 刺激的反应迟针, 出现心情压抑、焦虑、紧张, 疲劳 的现象。调查也发现, 他们均居住在相对集中的住舱 里, 室内经常有人休息, 所以经常灯光昏暗, 舱室空 气污浊。这些都说明, 不良环境引发了官兵心理贯乏, 日积月累出现了心理不稳定现象。根据调查进一步了 解, 发现他们一个共同的特点, 就是不喜欢参加集体 活动, 普遍认为“参加集体活动是一种被强迫的行为”。 对船上的广播反应不敏感, 对上级安排的任务也是应 付了事, 甚至出现将所属区域广播关闭的现象, 心理 排异现象严重。

\section{2. 当前我国远航船员思想管理现状}

对于管理者和一级组织而言而言, 最重要的是四 种主要功能: 控制、激励、情绪表达和信息[1],现阶 段我国远洋船员的思想管理方法上和制度上有如下 现状。

\section{1. 条令法规制度现状}

缺少针对性的条令条例法规制度, 舰员的管理工 作主要的法规依据为《中华人民共和国船员条例》和 《海事劳工公约》, 并没有专门对于人员思想管理指 导性的文件法规。李方圆[2]对于远洋船员的海事劳工 公约进行了总结, 我国无论是国内外还是海事部门, 都没有对于远洋航行人员思想管理的法规文件, 仅有 一些上级机关制定的行政管理条令。在各项管理条令 当中强调的船员专业技能要求和管理要求较多, 缺少 符合新时代的人文关怀, 这些管理条令法规对于船员 的心思想建设和心理健康问题不能达到提高和预防 的目的。

\section{2. 随船专业心理干预人员现状}

我国对于船员的思想管理多半依托于日常行政管 理的制度约束和随舰船医生的加强指导和心理疏导。 比较依靠经验和以往的惯性做法, 对于说教式的引导 教育往往收效甚微。例如我国海军最初几批执行亚丁 湾护航任务的加强军医, 在实际任务执行过程中, 出 现了对任务官兵和现阶段海军基层官兵的心理状况 并不是很掌握的情况, 最终心理干预收效甚微。

\section{3. 意识形态领域现状}

随着一批批95后, 00后加入军营船员队伍, 在给 各单位注入活力的同时, 带给各船意识形态领域的冲 击也慢慢凸现出来, 年轻船员思想活跃, 特别是一些 大学生, 文化程度较高, 有一些自己思维方式, 对于 人员的思想管理更是提出挑战, 一方面年轻船员从小 家庭富裕, 接受的教育环境不一样, 思想比较前卫, 同时受西方鼓吹的生活方式和文化侵蚀比较大, 也长 期习惯了网络信息时代带给他们的便利, 另一方面, 他们受一些诱惑性的价值理念影响, 加之长期出海, 能够获得的外界信息仅仅是卫星电视和电话, 很大程 度地抑制他们对外界社会信息的获取, 这就势必造成 许多船员谈论、抱怨上级的管理制度和生活工作，无 形中也影响着其他年龄段的舰员，与一些年纪较大的 舰员在思想上的代沟也更加放大了这种隐患, 最终导 致内部士气低沉，影响远航任务执行。

\section{3. 提升我国远洋船员思想管理的方法}

针对我国船员当前思想管理情况，运用各种管理 研究方法促进保健和激励作用, 审时度势地运用到船 员身上,特提出相应的对策措施, 以期提升思想管理水 平。

\section{1. 组织管理方法}

上级船运公司在任务开始前精准施策，多听取任 务船队的需求, 对于一些可去可不去的加强人员, 要 根据任务的需求实事求是地选派人员。对于随船加强 的人员, 首先应通过心理测试, 并选择一些有经验的 人员参与航行任务。通过多样风趣的文化活动组织, 点缀枯燥无味的航行生活。积极开展 “海上课堂”, 让 船员在整个航行任务过程中航行一路、学习一路、提 高一路。精心组织演讲活动, 让每名船员都能有机会 走上讲台, 讲述自己的心路历程和成长感悟, 每周休 息时间，组织卡拉OK大家唱，让大家在歌声中放飞 梦想, 释放心灵。

\section{2. 科学管理方法}

减少期望效应，正确运用激励作用，根据不同专 业岗位, 不同能力素质的船员, 针对他们的不同发展, 适时开展教育引导, 进一步提高船员思想认识, 做好 长期“吃大苦、耐大劳、严守纪”的心理准备，在潜意 
识中降低对于情绪表达和信息获取的期望值，矫正错 误心理预期, 为整体营造一个健康向上的工作环境, 从而减少负面情绪发生的概率。优化听觉环境, 适时 播放不同旋律的音乐，体育锻炼时播放节奏感强，充 满激情的音乐，就餐、就寝前播放节奏平缓、柔和的 音乐。优化视觉环境，根据不同的颜色会使人产生不 同的心理效应: 浅色使空间显得宽敞亮堂, 令人心情 舒畅; 深色作用则相反。及时更换老旧灯管, 使舱室 保证充足照明。优化嗅觉环境。整治舱室脏乱现象, 规范各房间物品摆放标准, 定期进行检查, 加强室内 通风, 及时消毒防疫。高质量的伙食对心理疏导极有 帮助。航行中要想方设法丰富膳食供应, 坚持好伙食 评议制度。精心调剂膳食, 坚持每餐有小菜有爽口菜, 保证三餐有变化, 每月举行集体生日会, 给过生日的 同志做蛋糕, 擀长寿面, 每周组织集体包包子、包饺 子, 既调剂了伙食花样, 又融洽了船员之间的关系。

\section{3. 加强人文关怀}

远航过程中, 工作强度高, 生活模式相对单调, 与亲人、外界联系少，容易对船员造成心理和生理的 影响。体能训练能丰富生活方式, 转移注意力, 调高 身体免疫力, 对船员适应远航生活, 完成航行任务有 较强的调节作用。将体能训练制度化, 利用各船的合 理场地例如甲板, 健身房等活动场所, 同时将人员科 学划分, 规定每人每日锻炼必须在 30 分钟以上。适 时组织“甲板趣味运动会”, 在项目设置上, 不仅有水 中憋气、平板支撑等个人单项, 还可设置接力跑、团 结一致向前进等趣味性较强的团体项目, 让船员在团 结互助中增进友谊。在执行任务前, 可以适时组织船 员采用 SCL-90 量表[3]问卷调查、茶话会等方式, 了 解船员的所思所想, 诉求困扰。在任务中, 对于年轻 船员特别是缺少经验的新船员要加强关心和监督的 力度。远航结束后, 及时收集船员所反馈的思想管理 问题, 并及时反馈上级为每一次成功返航奠定基础和 好的经验做法。

\section{4. 总结}

商船远航任务完成的顺利与否, 关键在于人员思 想, 而解决这些问题仅靠单一制度或政治部门教育是 远远不够的, 必须调动各方面力量, 形成整体合力。 对于之前所沿袭下来的惯性管理思维, 必须要转变, 更多地创新管理方法, 体现人文关怀, 要在理解人、 尊重人的基础上教育人、引导人。要多一些换位思考、 设身处地、推心置腹, 为船员着想, 切实做到尊重船 员思想, 理解船员处境、愿望、心理和性格以达到合 理，合法的管理目的。

\section{致谢}

本论文在杜荔红教授的悉心指导下完成, 杜教授 渊博的专业知识, 严谨的治学态度, 精益求精的工作 作风, 海人不倦的高尚师德深深感染着我, 本次论文
从定题到完成, 都是在杜教授悉心指导下完成的, 遇 到的问题, 都在杜教授的指导下得以解决, 所以在此, 在此向杜教授道一声：谢谢您！

\section{REFERENCES}

[1] W.G.Scott and T.R.Mitchell, Organization Theory: A Structural and behavioral Analysis(Homewood, IL:Richard D.Irwin,1976).

[2] Li Fangyuan, Liu Shengyou, Li Wei. Analysis on the mental health construction of Chinese ocean-going seafarers [J]. China Maritime Affairs, 2018(05):38-40.

[3] Wu Yuanhe, Zhang Bei. Cultivation of seafarers' professional psychological adaptability [J]. Maritime Education Research, 2005(02):96-99. 